\title{
Editorial
}

\section{Gamma-Ray Burst in the Swift/Fermi Era and Beyond}

\author{
WeiKang Zheng $\mathbb{D}^{1},{ }^{1}$ Takanori Sakamoto $\mathbb{D}^{2},{ }^{2}$ Yuji Urata, ${ }^{3}$ and Shashi B. Pandey $\mathbb{D}^{4}$ \\ ${ }^{1}$ Department of Astronomy, University of California, Berkeley, CA 94720-3411, USA \\ ${ }^{2}$ Department of Physics and Mathematics, Aoyama Gakuin University, Sagamihara, Kanagawa 252-5258, Japan \\ ${ }^{3}$ Institute of Astronomy, National Central University, Chung-Li 32054, Taiwan \\ ${ }^{4}$ Aryabhatta Research Institute of Observational Sciences, Manora Peak, Nainital 263002, India \\ Correspondence should be addressed to WeiKang Zheng; zwk@astro.berkeley.edu
}

Received 20 December 2016; Accepted 20 December 2016; Published 1 February 2018

Copyright (C) 2018 WeiKang Zheng et al. This is an open access article distributed under the Creative Commons Attribution License, which permits unrestricted use, distribution, and reproduction in any medium, provided the original work is properly cited.

The Swift and Fermi satellites have been observing gammaray bursts (GRBs), which are short-lived and intense flashes of gamma-rays associated with the death/explosion of massive stars and/or compact binary mergers, for nearly a decade in space since their launch in 2004 and 2008, respectively. Our understanding of GRB physics, though still far from complete, has been revolutionized thanks to their outstanding observations, as well as the ongoing ground follow-up observations. In addition to a previously published special issue, "Gamma-Ray Bursts in the Swift and Fermi Era," which discussed GRB work related to the Swift and Fermi missions, here we continue collecting reviews and research articles on GRBs and their afterglows.

In total, five reviews and one research article are published in this special issue. The review article by M. Tanaka summarizes the current understanding of kilonovae/macronovae related to the GRBs. In comparison, Z. Cano et al. present an up-to-date review of progress on the connection between supernovae and long-duration GRBs. Next, the review article by $M$. Dainotti et al. discusses various GRB correlations during the prompt phase and their use as redshift estimators and possibly as cosmological tools. In contrast, the review by J. J. Geng and Y. F. Huang focuses on the GRB afterglow phase, discussing the diverse features of the multiwavelength afterglows of GRBs. One other article by M. Jelinek et al. presents a decade of optical follow-up observations of GRBs by the BOOTES telescope in Spain. Lastly, P. Chandra discusses GRBs from the radio perspective, which is very important for studies of GRBs related to their calorimetry, reverse-shock emission, and circumburst environments.
Overall, this volume extends the discussions of the previously published special issue, "Gamma-Ray Bursts in the Swift and Fermi Era," and covers more topics about GRBs from gamma-ray to radio wavelengths. These are the most updated summaries of progress on GRB studies during the Swift and Fermi mission periods. We hope these two special issues provide useful information for GRB researchers, helping to lead to new results and progress even beyond the Swift and Fermi era.

\section{Acknowledgments}

We sincerely thank the authors and referees for all of their efforts.

\author{
WeiKang Zheng \\ Takanori Sakamoto \\ Yuji Urata \\ Shashi B. Pandey
}



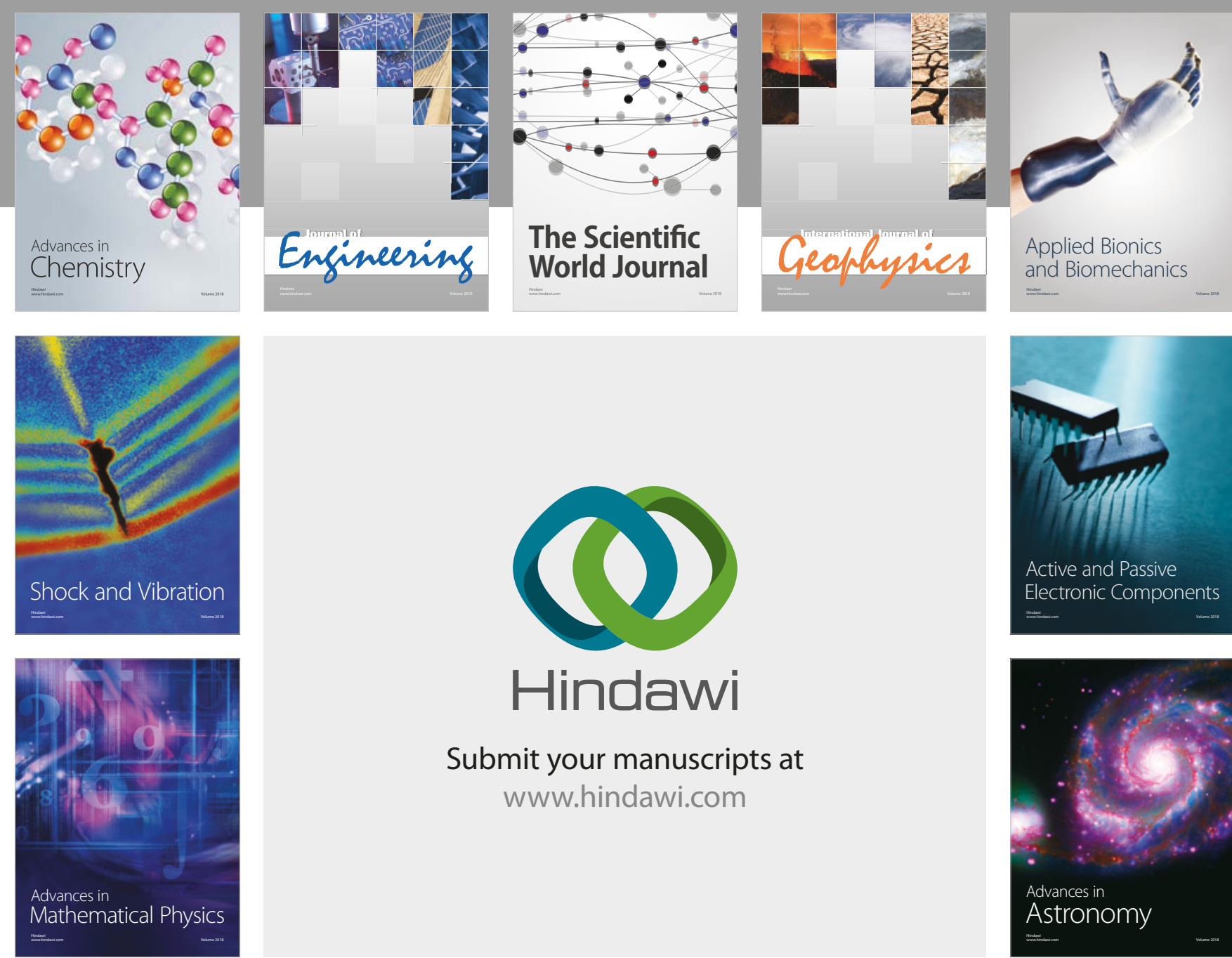

Submit your manuscripts at

www.hindawi.com

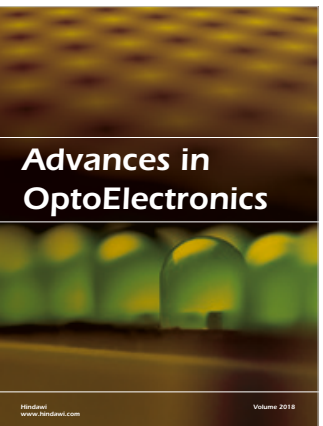

\section{Rotcting Machinery}
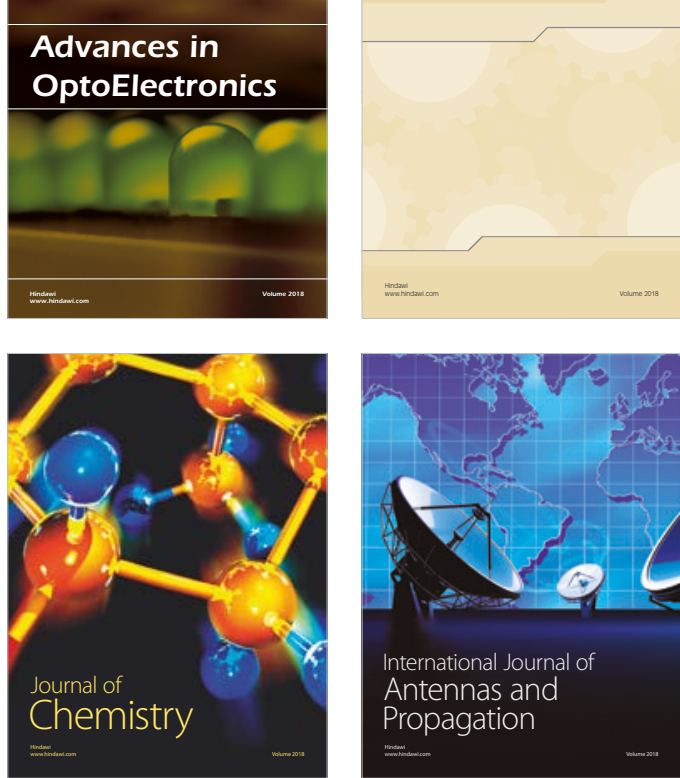

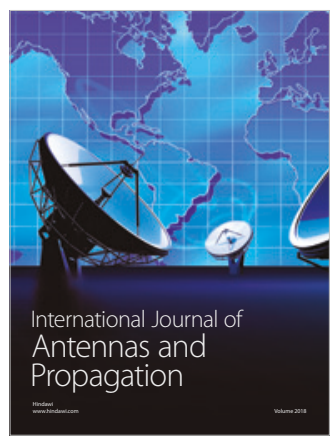

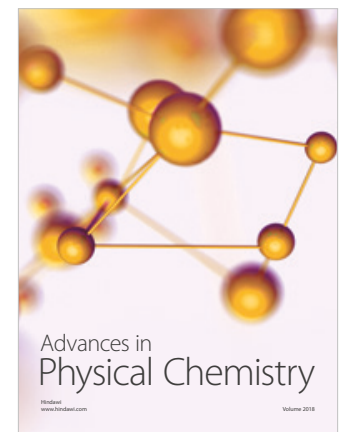

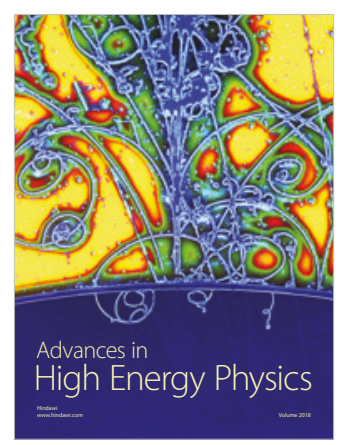

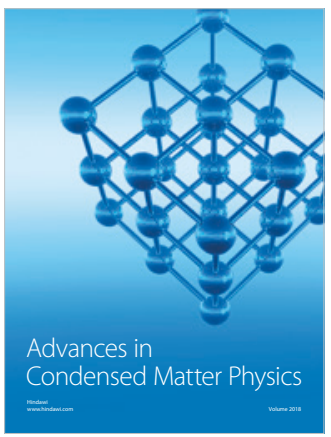

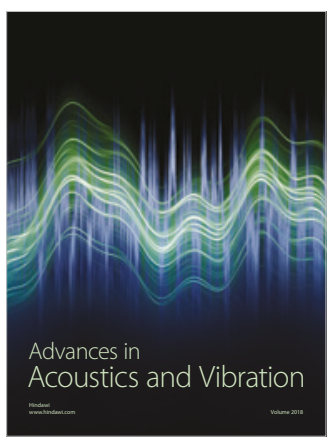

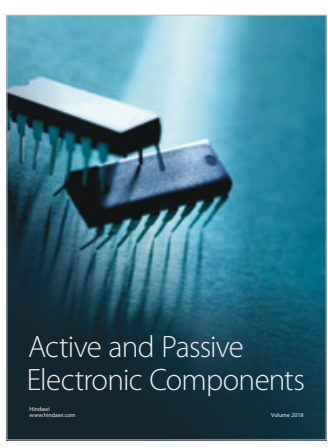
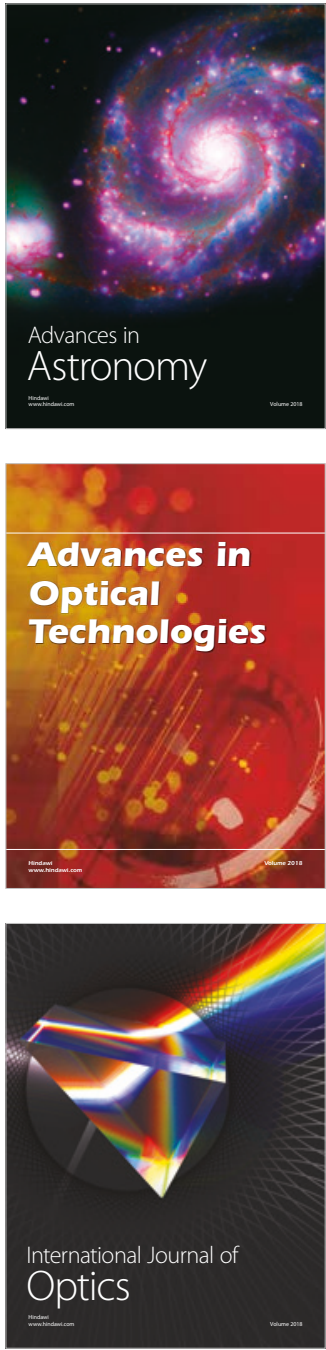